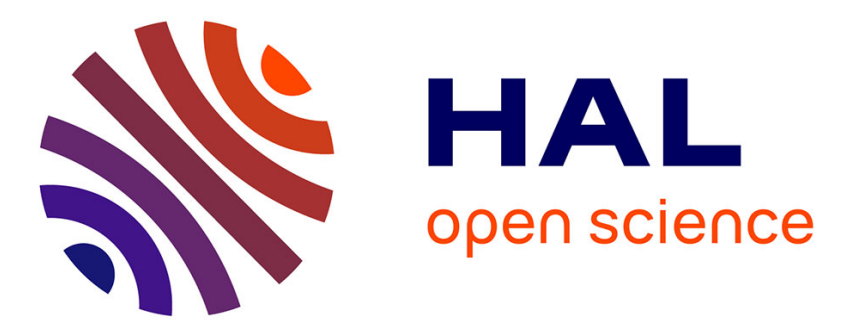

\title{
Measurement System of Reducing Temperature Fluctuation of Thermostat Bath for Calibrating Thermocouple
}

Min Zhang, Feixia Liang, Yue Xie, Ruguo Huang, Haitao Yuan, Jiahua Lu

\section{- To cite this version:}

Min Zhang, Feixia Liang, Yue Xie, Ruguo Huang, Haitao Yuan, et al.. Measurement System of Reducing Temperature Fluctuation of Thermostat Bath for Calibrating Thermocouple. 8th International Conference on Computer and Computing Technologies in Agriculture (CCTA), Sep 2014, Beijing, China. pp.603-609, 10.1007/978-3-319-19620-6_67 . hal-01420277

\section{HAL Id: hal-01420277 \\ https://hal.inria.fr/hal-01420277}

Submitted on 20 Dec 2016

HAL is a multi-disciplinary open access archive for the deposit and dissemination of scientific research documents, whether they are published or not. The documents may come from teaching and research institutions in France or abroad, or from public or private research centers.
L'archive ouverte pluridisciplinaire HAL, est destinée au dépôt et à la diffusion de documents scientifiques de niveau recherche, publiés ou non, émanant des établissements d'enseignement et de recherche français ou étrangers, des laboratoires publics ou privés.

\section{(c)(1)}

Distributed under a Creative Commons Attribution| 4.0 International License 


\title{
Measurement System of Reducing Temperature Fluctuation of Thermostat Bath for Calibrating Thermocouple
}

\author{
Min Zhang ${ }^{\mathrm{a}}$, Feixia Liang ${ }^{\mathrm{b}}$, Yue Xie ${ }^{\mathrm{c}}$, Ruguo Huang ${ }^{\mathrm{d}}$, Haitao Yuan ${ }^{\mathrm{e}}$, Jiahua $\mathrm{Lu}^{\mathrm{f}}$ \\ College of Food Science and Technology, Shanghai Ocean University, Shanghai 201306, \\ China \\ a zhangm@shou.edu.cn, ${ }^{\mathrm{b}}$ 1375176575@qq.com, ${ }^{\mathrm{c}} 313929785 @ q q . c o m,{ }^{\mathrm{d}}$ \\ 895251475@qq.com, ${ }^{\mathrm{e}} 784991342 @ q q . c o m,{ }^{\mathrm{r}}$ lujiahua.home@yahoo.com.cn
}

\begin{abstract}
Based on the periodic unsteady state heat conduction theory, a new measurement system of reducing temperature fluctuation of thermostat bath was developed in order to obtain a liquid environment with uniform and constant temperature controlled for the measurement requirements of calibrating thermocouple. The experimental results show that the temperature stability in this measurement system is superior to that in traditional system. The measurement system had the advantage of calibrating multipoint thermocouple at the same time and completing the data acquisition and control automatically.
\end{abstract}

Keywords: Temperature fluctuation, Thermostat bath, Thermocouple, Calibrate

\section{Introduction}

Low-temperature thermostat bath is essential laboratory equipment in food, biological, chemical scientific research and industrial applications[1]. It is mainly used to provide liquid controlled environment with uniform and constant temperature. It can be used as heating or cooling source or secondary heating or cooling source[2-3]. It is often used as thermocouple calibration equipment in the measurement of food thermal properties. So the performance of low-temperature thermostat bath have a directly impact on the reliability and accuracy of thermocouple calibrating [4]. However, there is a defect of bigger temperature fluctuations and consistent unsteady temperature in moderate low-temperature thermostat bath because the temperature of liquid in thermostat bath is affected by compression engine running and fluid convection heat transfer. As a result, it is difficult to get high precision of temperature calibration of thermocouple which is put in the liquid[5-8]. Especially in the process of calibrating multiple thermocouples at the same time, multipoint stable temperature environment is essential. To solve these problems, on the basis of the traditional thermostat bath, a new measurement system of reducing temperature fluctuation of thermostat bath was developed and T-type thermocouple temperature calibration value had been analyzed. 


\section{Principle}

Based on the Seebeck effect, temperature was measured by thermocouples[9]. To reduce errors of temperature measurement system, the temperature dividing should be calibrated due to different thermocouples materials which would cause difference of thermoelectric properties and allowable deviation [7]. The temperature deviation would be revised according to the measured temperature value of standard thermometer and thermocouple in the same liquid environment with uniform and constant temperature controlled which is provided by low-temperature thermostat bath. The thermocouples and standard thermometer are directly put into a lowtemperature thermostat bath. But the inner liquid would produce cyclical fluctuations of heating and cooling and then it would pass to measured surface. The reason is that temperature control is achieved by means of hot and cold offset.

According to the theory of heat transfer[10], the measured object can be considered as a homogeneous semi-infinite body. The temperature field in boundary conditions of periodic change for semi-infinite homogeneous object, can be descripted by nonsteady-state heat conduction differential equation [11] :

$$
\frac{\partial t}{\partial \tau}=\alpha \frac{\partial^{2} t}{\partial x^{2}} \quad \text { ( } 1 \text { ) }
$$

The boundary conditions can be considered to be a harmonic. Then the temperature changes in the surface of object can be obtained as cosine function:

$$
\theta=A_{w} \cos \frac{2 \pi}{T} \tau
$$

By separation of variables method, equation ( 1 ) ( 2 ) can be integrated and the internal temperature distribution of object is as follow:

$$
\theta(x, \tau)=A_{w} \exp \left(-x \sqrt{\frac{\pi}{\alpha T}}\right) \cos \left(\frac{2 \pi}{T} \tau-x \sqrt{\frac{\pi}{\alpha T}}\right) \quad(3)
$$

Where $t$ is object temperature (K), $\tau$ is heating time (s), $\alpha$ is thermal diffusion coefficient $\left(\mathrm{m}^{2} / \mathrm{s}\right), x$ is distance from the surface $(\mathrm{m}), \theta$ is instantaneous surplus temperature on the surface of the $\operatorname{object}(\mathrm{K}), \theta(x, \tau)$ is instantaneous surplus temperature inside the $\operatorname{object}(\mathrm{K}), A_{w}$ is temperature fluctuation on the surface $(\mathrm{K})$, $T$ is the cycle of wave period (s).

By equation ( 2 ) and ( 3 ), the surface temperature and the internal temperature will be periodic changes over time. But the fluctuation amplitudes of internal temperature is less than that of surface temperature. Meanwhile, the amplitude decreases greatly with increase of $\mathrm{x}$. The extent of the amplitude attenuation can be expressed:

$$
v=\exp \left(-x \sqrt{\frac{\pi}{\alpha T}}\right)
$$

It shows that internal wave amplitude decreases with the deepening of the temperature wave. It is one of the important characteristics of periodic heat conduction. That is to say, the temperature wave amplitude increases gradually as the surface distance 
attenuation. From the theory, reducing the test ambient temperature fluctuation degree can ensure a liquid environment with uniform and constant temperature controlled.

\section{Test System and Method}

\subsection{Test System}

As shown in Figure 1, thermocouple calibration measurement system is based on the above principles. The test device includes low-temperature thermostat bath, temperature balance outer pipe, temperature balance inner pipe, blender, insulation cover, insulated container, standard platinum resistance, thermocouple, data acquisition device and computer.

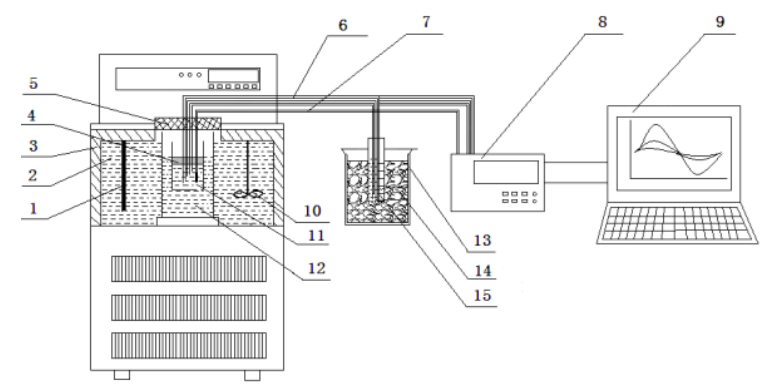

Fig. 1. Schematic diagram of measurement system

1. Heating tuber 2. Thermostat bath 3. Insulation layer 4. insulation cover 5.Outer pipe insulation cover 6. Thermocouple 7.Standard platinum resistance 8. Data acquisition device 9. Computer 10. Blender 11. Balance outer pipe 12 balance inner pipe 13.Insulated container 14. Ice-water mixture 15 . Heat transfer oil

The outer pipe and inner pipe of temperature balance are located in the thermostat bath. Flowing medium level within thermostat bath is above temperature balance outer pipe. Flowing medium level within temperature balance outer pipe is higher than temperature balance inner pipe. An anti-mutual contact device is set between two pipes and make inner pipe fix in the middle of outer pipe. The anti- mutual contact device is not touched the bottom and sides wall of temperature balance outside tube. A supporting insulation cover is used on the outer pipe and inner pipe of temperature balance. A calibrated standard platinum resistance thermometer and several groups of non- touching thermocouples were put in the temperature balance inner pipe at the same time. Platinum resistance thermometer and thermocouples fixed by stents located in the inner pipe nozzle, and not touched bottom and sides wall of temperature balance pipe. Then temperature data of standard platinum resistance thermometer and thermocouples were collected and transferred to computer by FLUKE data acquisition instrument. 


\subsection{Standard Test Method}

DC2006 low-temperature thermostat bath was used for this work. The flume dimension was length $250 \mathrm{~mm}$, width 200 and depth $200 \mathrm{~mm}$. The temperature range was $20-95^{\circ} \mathrm{C}$. With water as a working fluid, the heating system is made of L Type stainless steel heating pipe. The heating end was at the bottom of thermostat bath. The evaporator in refrigeration system was made of copper pipe, which was placed directly in the sink in order to increase the cooling rate. $T$ copper-constantan thermocouples with diameter $0.5 \mathrm{~mm}$ were selected. The reference terminals were immersed in transformer oil surrounded by ice-water mixture. The temperature balance pipes were made of high borosilicate glass material. The outer pipe was external diameter $100 \mathrm{~mm}$, depth $100 \mathrm{~mm}$ and wall thickness $3 \mathrm{~mm}$, while the inner pipe external diameter $75 \mathrm{~mm}$, depth $100 \mathrm{~mm}$ and wall thickness $3 \mathrm{~mm}$. Due to the presence of glass containers, the distance between thermocouple and fluid walls was increased in the process of calibrating the thermocouple, which could effectively prevent temperature fluctuation caused by heat convection [12] .

First, water was poured into the thermostat bath with the outer pipe and inner pipe of temperature balance. The water level of the thermostat bath was above the water level of the temperature balance outer pipe, and the water level in the outer pipe was higher than in inner pipe. Secondly, T type thermocouples and calibrated standard platinum resistance thermometer were fixed in the centre of the temperature balance inner pipe by support and kept isolated and out of contact each other. Thirdly, insulated cover was sealed. Finally, the other ends of $\mathrm{T}$ type thermocouples and platinum resistor were connected to FLUKE Data collecting instrument into a computer.

Temperature data were collected every 30 seconds during testing. The changes of temperature were observed in real-time detection. The temperature stability data could be collected when adjusting to the required temperature [13]. T type thermocouple and calibrated standard platinum resistance thermometer could be compared and corrected at the same temperature. Every degree of temperature value of $\mathrm{T}$ type thermocouple was calibrated in turn and was contrasted directly place the thermocouple in water and only place the thermocouple in temperature balance inner tube.

\section{Results and Discussion}

\subsection{Temperature Fluctuations}

Fig.2, Fig.3 and Fig.4 showed T type thermocouple temperature test case when liquid temperature in the thermostat bath was set to $20^{\circ} \mathrm{C}$, respectively. 


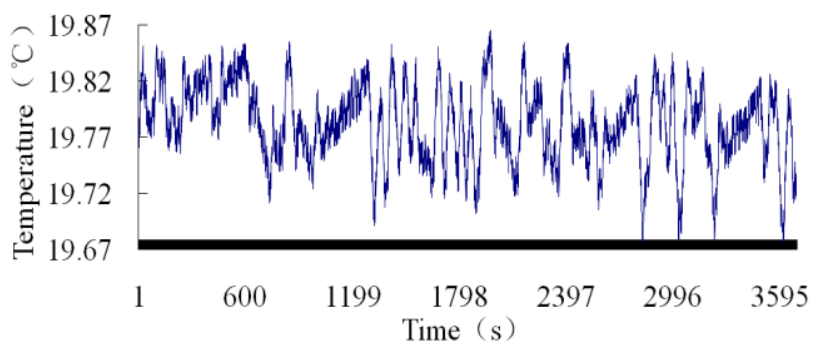

Fig. 2. Temperature test curve of thermocouple put in water

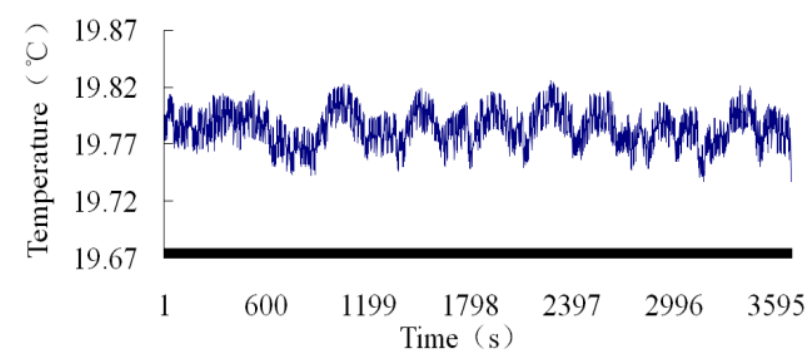

Fig. 3. Temperature test curve of thermocouple put in temperature balance tube Water

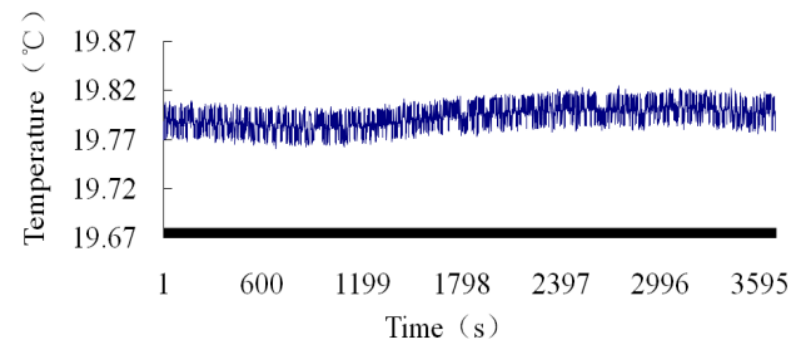

Fig. 4. Temperature test curve of thermocouple put in measurement system

As can be seen from the diagram, temperature wave amplitude of $\mathrm{T}$ Thermocouple in contact with fluid directly was greater than other two conditions. Calibration temperature wave amplitude of the T type thermocouple in contact with fluid directly was $\pm 0.075^{\circ} \mathrm{C}$. Calibration temperature wave amplitude of only increasing outer pipe is $\pm 0.03{ }^{\circ} \mathrm{C}$, Calibration temperature wave amplitude of simultaneously increasing outer pipe and inner pipe was $\pm 0.02^{\circ} \mathrm{C}$. The temperature stability raised $73.3 \%$ and $33.3 \%$ than the previous two conditions, respectively.

It was shown that the thermal resistance of the glass could prevent temperature fluctuation causing by convection. Test results were in accord with the analytical solutions for natural convection boundary layer equations by Pohlhausen and research results of Magyari and Myers [14-15] . 


\subsection{Thermocouple Calibration}

As shown in Table 1, test results of multipoint $\mathrm{T}$ type thermocouple under the conditions of 5- $50{ }^{\circ} \mathrm{C}$, which happened in test device with the temperature balance outer pipe and inner pipe.

Table 1. Calibration result of multipoint thermocouple

\begin{tabular}{|c|c|c|c|c|c|c|}
\hline \multirow{2}{*}{$\begin{array}{l}\text { Thermosta } \\
\text { t setting } \\
\text { temperatur } \\
\text { e }\left({ }^{\circ} \mathrm{C}\right)\end{array}$} & \multicolumn{5}{|c|}{ Temperature fluctuations of thermocouple test point $\left({ }^{\circ} \mathrm{C}\right)$} & \multirow{2}{*}{$\begin{array}{l}\text { Temperature } \\
\text { fluctuations in } \\
\text { average }\left({ }^{\circ} \mathrm{C}\right)\end{array}$} \\
\hline & 1 & 2 & 3 & 4 & 5 & \\
\hline 5 & \pm 0.02 & \pm 0.01 & \pm 0.02 & \pm 0.01 & \pm 0.02 & \pm 0.02 \\
\hline 10 & \pm 0.02 & \pm 0.02 & \pm 0.02 & \pm 0.02 & \pm 0.02 & \pm 0.02 \\
\hline 20 & \pm 0.02 & \pm 0.02 & \pm 0.02 & \pm 0.02 & \pm 0.02 & \pm 0.02 \\
\hline 30 & \pm 0.02 & \pm 0.02 & \pm 0.03 & \pm 0.02 & \pm 0.03 & \pm 0.02 \\
\hline 40 & \pm 0.03 & \pm 0.03 & \pm 0.03 & \pm 0.03 & \pm 0.03 & \pm 0.03 \\
\hline 50 & \pm 0.03 & \pm 0.03 & \pm 0.03 & \pm 0.03 & \pm 0.03 & \pm 0.03 \\
\hline
\end{tabular}

As can be seen from the Table 1, average temperature fluctuation was $\pm 0.02^{\circ} \mathrm{C}$ and $\pm 0.03^{\circ} \mathrm{C}$ in the range of $5-30^{\circ} \mathrm{C}$ and $40-50^{\circ} \mathrm{C}$, respectively. What is more, the experiments were shown no uniform temperature field only occurred in closing to the wall surface of heat transfer in convection heat transfer. The temperature balance inner pipe fixed in outer pipe and outer pipe fixed in insulated cabinet. The presence of glass container increased distance between thermocouple and wall surface in calibration process, attenuated temperature wave amplitude, effectively prevented temperature fluctuations caused by convection heat transfer. The purpose of precise calibration was achieved.

\section{Conclusions}

The designed measurement system device included low-temperature thermostat bath, temperature balance outer pipe, temperature balance inner pipe, blender, insulation cover, insulated container, standard platinum resistance, thermocouple, data acquisition device, computer and so on. T type thermocouple under the conditions of $5-50{ }^{\circ} \mathrm{C}$ was calibration with average temperature fluctuation $\pm 0.02{ }^{\circ} \mathrm{C}$ and \pm $0.03^{\circ} \mathrm{C}$ in the range of $5-30^{\circ} \mathrm{C}$ and $40-50{ }^{\circ} \mathrm{C}$, respectively. It is simple, convenient and reliable. There will be a bright prospect for determining temperature in scientific research and engineering application. 


\section{Acknowledgment}

Funds for this research was provided by the Natural Science Foundation of China, Grant No 31371526.

\section{References}

1. Andrea Merlone, Luigi Iacomini, Antonio Tiziani et al. A liquid bath for accurate temperature measurements[J].Measurement, $2007,40(4): 422-427$.

2. Wu Jiangtao, Liu Zhigang, Wang Fengkun, et al. A new research of the high-precision thermostatic bath at low temperature for fluid thermal physical measurement [J]. Journal of $\mathrm{Xi}^{\prime}$ an Jiao tong University, $2004,38(5)$ : 504-507.

3. Andrea Merlone, Marcarino P., Iacomini, L. et al. A liquid bath for accurate platinum resistance thermometers calibrationat IMGC, the 9thInternational Symposium on Temperature and Thermal Measurements in Industry and Science. 2004, 929-933.

4. Zhang P., Y.X. Xu, R.Z. Wang, et al. Fractal study of the fluctuation characteristic in the calibration of the cryogenic thermocouples[J]. Cryogenics, 2003, 43(1):53-58.

5. Fan $\mathrm{Hu}, \mathrm{ZeShao}$ Chen, Dawei Luo, et al. A research of the precise low-temperature thermostat bath for refrigerants PVT Development for experiment [J]. Chinese Journal of scientific instrument, 2002, 23(4): 414-416.

6. Abdelaziz Y.A., Megahed F.M., Halawa M.M.. Stability and calibration of platinum/palladium thermocouples following heat treatment [J]. Measurement, 2004, 35(4) :413-420.

7. Laura Villafañe, Guillermo Paniagua. Aero-thermal analysis of shielded fine wire thermocouple probes [J]. International Journal of Thermal Sciences, 2013, 65(3):214-223.

8. Utpal Sarma, P.K. Boruah. Design and development of a high precision thermocouple based smart industrial thermometer with on line linearisation and data logging feature [J]. Measurement, 2010, 43(10):1589-1594.

9. Danisman K., Dalkiran I., Celebi F.V.. Design of a high precision temperature measurement system based on artificial neural network for different thermocouple types [J]. Measurement, 2006, 39(8): 697-700.

10.Holman J. P., Heat Transfer,10th Ed. McGraw-Hill, New York, 2002.

11.Yang Shiming, Tao Wenquan. Heat transfer [M]. The Fourth Edition. Beijing: higher education press, 2007.

12.Nouanegue H., Muftuoglu A., Bilgen E.. Conjugate heat transfer by natural convection, conduction and radiation in open cavities. International Journal of Heat and Mass Transfer[J], 2008, 51(25-26): 6054-6062.

13.Yu Wenfang, Zhao Wenmin. The computer measurement and control system based on agilent34970A Data acquisition instrument [J]. Automation instrumentation, 2004, 10: 4648.

14.Magyari E.. Backward boundary layer heat transfer in a converging channel[J]. Fluid Dynamics Research, 2007, 39(6): 493-504.

15.Myers T.G.. An approximate solution method for boundary layer flow of a power law fluid over a flat plate[J]. International Journal of Heat and Mass Transfer, 2010, 53(11-12): 23372346. 